\title{
GMR
}

\section{Association of MEF2A gene 3'UTR mutations with coronary artery disease}

\author{
X.C. Huang and W. Wang \\ Department of Clinical Laboratory of Wuhan Puai Hospital, Wuhan, China \\ Corresponding author: X.C. Huang \\ E-mail: Xinci_Huang@163.com \\ Genet. Mol. Res. 14 (3): 11073-11078 (2015) \\ Received May 11, 2015 \\ Accepted July 13, 2015 \\ Published September 21, 2015 \\ DOI http://dx.doi.org/10.4238/2015.September.21.20
}

ABSTRACT. Association of variants in the myocyte enhancer factor $2 \mathrm{~A}$ (MEF2A) gene and the risk of coronary artery disease (CAD) has drawn much attention but remains controversial. We hypothesized that the 3'-untranslated region (3'-UTR) of this gene could harbor functionally relevant nucleotide changes. Here, we assessed the association between single nucleotide polymorphisms (SNPs) in the 3'-UTR of MEF2A and CAD in the Chinese Han population. A case-control study of 236 CAD patients and 278 controls was carried out. The four target SNPs were genotyped using a multiplex PCR-ligase detection reaction method. Logistic regression under three genetic models was used to analyze the association between target SNPs and the risk of CAD. Associations were detected between two SNPs (rs325380, rs897074) and CAD; however, after Bonferroni's correction, these associations were not deemed significant. A further haplotype study indicated that a 'TA' haplotype carrier of rs325380-rs325381 was associated with CAD risk. Our study thus indicates that variants in the 3 '-UTR of MEF2A are associated with CAD in a Chinese Han population.

Key words: Coronary artery disease; Single nucleotide polymorphism; MEF2A; Gene; 3'-UTR 


\section{INTRODUCTION}

Over the last decade, coronary artery disease (CAD) has become a major cause of death and disability in China. A recent study indicates that CAD is a result of both genetic and environmental effects (Kangas-Kontio et al., 2010). In line with this, myocite enhancer factor 2A (MEF2A) gene deficiency might lead to vascular endothelium abnormalities, which could precipitate atherogenesis in the presence of other CAD risk factors. In 2003, Wang et al. reported that the MEF2A gene was associated with CAD/MI in a single large family of Scandinavia ancestry. Thereafter, many case-control studies attempted to investigate the explicit effects of MEF2A on CAD (Guella et al., 2009; Elhawari et al., 2010; Juszczuk-Kubiak et al., 2012; Liu et al., 2012; Dai et al., 2010, 2013; Foroughmand et al., 2014). However, the results have been inconsistent.

The 3'-untranslated region (3'-UTR) is a key segment of mRNAs that control gene expression at the post-transcriptional level due to its impact on adenylation, mRNA stability/degradation, nuclear export, subcellular localization, and translation efficiency (Kuersten and Goodwin, 2003; Shyu et al., 2008). Chen et al. (2006a, b) performed systematic studies on disease-associated polymorphisms in the 3'-UTR of human protein-coding genes and revealed a correlation between the variants' functions and alterations in secondary structure. Furthermore, in 1997, Black et al. revealed that the 3'-UTRacts as a post-transcriptional repressor of MEF2A protein expression during the differentiation of muscle cells. To our knowledge, there is no description of variants in the MEF2A 3'-UTR associated with CAD in the Chinese Han population.

In the present case-control study, we attempted to determine whether variants in the 3'UTR of the MEF2A gene are associated with vulnerability to CAD.

\section{MATERIAL AND METHODS}

\section{Subjects}

This study was conducted between September 2012 and June 2014 in Wuhan Puai Hospital. All patients were confirmed to have CAD by angiography. The criteria of CAD diagnosis were listed as: at least one of the major coronary arteries, including the left anterior descending, left circumflex or right coronary artery, displayed at least $50 \%$ organic stenosis. A total of 236 CAD patients were consecutively recruited. At the same time, we recruited 278 control outpatient subjects who were excluded as suffering from CAD by computed tomography angiography or magnetic resonance angiography, or who underwent regular health examinations during the same time. The unaffected controls chosen from the out patients were appraised by questionnaires, clinical history, and electrocardiography to exclude CAD. Those control subjects with symptoms or signs of other atherosclerotic vascular diseases, abnormal electrocardiogram, or chronic liver and kidney disease were excluded. All subjects enrolled in this study were of Han Chinese ethnicity and residing in or near Hubei Province region. The study protocol was approved by the Ethical Committee of Wuhan Puai hospital (Identification code: 2012-009-01 approved on April 12, 2012). Informed consent was provided by each participant.

\section{Single nucleotide polymorphism (SNP) selection and genotyping}

We executed a search on website (http://www.ncbi.nlm.nih.gov/projects/SNP/) and found that the MEF2A gene can encode a wide range of 3 '-UTRs. We chose rs325380, rs325381, rs325383, and rs897074 as tag-SNPs based on MAF values $\geq 0.05$ in CHB (Han Chinese in 
Beijing, China). The genomic DNA was extracted using the PAX gene Blood DNA kit (Qiagen, Hilden, Germany) according to the product instructions. Genomic DNA was genotyped using PCRbased ligation detection reaction (PCR-LDR) method. The PCR primers for the four loci (rs897074, rs325383 rs325381, and rs325380) were 5'-CAGAACGATGCAGCTGGTTA-3' (forward) and 5'-TTCAAAATCCAAGCTGAGGG-3' (reverse) for rs897074; 5'-CCCCCAAATTACGTTCCTTT-3' (forward) and 5'-GCCATGAGAACAGAACCTCC-3' (reverse) for rs325383; 5'-CACCCACATCCACATCT CTG-3' (forward) and 5'-AGGAAGGACAGCTGTTGGAA-3' (reverse) for rs325381; 5'-CTGGACTTTGT TGCCATCCT-3' (forward) and 5'-GGAATGAAAAAGGAGAGGGC-3' (reverse) for rs 325380 . The PCRs were performed by ABI 9600 (Applied Biosystems, Foster City, CA, USA) in a total volume of $20 \mu \mathrm{L}$, containing $1 \mu \mathrm{L}$ genomic DNA, 1.5 $\mu \mathrm{L}$ 10X PCR buffer (TaKaRa, Japan), $0.15 \mu \mathrm{m}$ of each primer (Sangon, China), $0.2 \mu \mathrm{m}$ dNTP (Sangon, China), $0.25 \mu \mathrm{L}$ Taq DNA polymerase (Qiagen $\mathrm{GmbH}$, Hilden, Germany), and $\mathrm{ddH}_{2} \mathrm{O}$. The cycling parameters were: $95^{\circ} \mathrm{C} 5 \mathrm{~min}, 35$ cycle $\left(94^{\circ} \mathrm{C}-\right.$ $30 \mathrm{~s}, 60^{\circ} \mathrm{C}-30 \mathrm{~s}, 72^{\circ} \mathrm{C}-50 \mathrm{~s}$ ), with a final extension at $72^{\circ} \mathrm{C}$ for $2 \mathrm{~min}$. For each PCR product, the ligation reaction was performed in a final volume of $10 \mu \mathrm{L}$, including $2 \mu \mathrm{L}$ PCR product, $1 \mu \mathrm{L} \mathrm{10X} \mathrm{Taq}$ DNA ligase buffer, $0.02 \mu \mathrm{M}$ probe mixture, $5 \cup$ Taq DNA ligase (New England Biolabs, Beverly, MA, USA), and $6 \mu \mathrm{L} \mathrm{H}_{2} \mathrm{O}$. The probe was shown in Table 1. The LDRs were cycled as follows: 35 cycles at $94^{\circ} \mathrm{C}$ for $30 \mathrm{~s}$ and $54^{\circ} \mathrm{C}$ for $4 \mathrm{~min}$. The LDR products were analyzed on ABI 377 DNA sequencer (Applied Biosystems, USA). Five percent of the samples were randomly selected for genotyping twice by different researchers for quality control, and the results were $100 \%$ concordant.

Table 1. Sequences of the primers and probes used SNP genotyping.

\begin{tabular}{ll}
\hline Probe names & Sequence $\left(5^{\prime}-3^{\prime}\right)$ \\
\hline rs325380-FAM & P-ACAATGCTAAAGGTTGTTGTAAATTTTTTTTTTTTTTTTTTTTTTTTTT-FAM \\
rs325380-T & TTTTTTTTTTTTGAACTTTTTATCAAATGGTGA \\
rs325380-G & TTTTTTTTTTTTTTTGAACTTTTTATCAAATGGTGC \\
rs325381-FAM & P-GGGGACGACGCTAATGGTGTTGCTTTAGAACCTTTTTTTTTTT-FAM \\
rs325381-T & TTTTTTTTTTTTGGAAACCAGCCTAGAGA \\
rs325381-A & TTTTTTTTTTTTTTTTGGAACCAGCCTAGAGT \\
rs325383-FAM & P-TTTTCCTCATCTGCTGTTTTTTTTTTTTTTTTTTTT-FAM \\
rs325383-C & TTTTTTCCAAATTACGTTCCTTTTGACG \\
rs325383-T & TTTTTTTTCCAAATTACGTTCCTTTTGACA \\
rs897074-FAM & P-GGTCCTTGGCATGACTCTTGCCATTTTTTT-FAM \\
rs897074-C & TTTAGAAACACTCTTAGGGTGCC \\
rs897074-T & TTTTTTAGAAACACTCTTAGGGTGCT \\
\hline
\end{tabular}

\section{Statistical analysis}

Hardy-Weinberg equilibrium and allele frequencies were confirmed by chi-square tests. The association between target SNPs and the risk of CAD with the adjustment for age, gender, and smoking status was analyzed by unconditional logistic regression under three genetic models including codominant, dominant and recessive models. Bonferroni's correction was applied, since we evaluated multiple SNPs and genetic models. To further analyze the association between haplotypes and CAD risk, linkage disequilibrium (LD) blocks were constructed by Haploview software using SNP genotyping data. The statistical analysis was carried out by SPSS 16.0 (SPSS, Inc., Chicago, USA) and SNPStats online software (http://bioinfo.iconcologia.net/SNPstats).

\section{RESULTS}

Two-hundred thirty-eight individuals with CAD were enrolled in the study. The sample 
comprised 158 (66.9\%) men in case group. The controls were selected from persons who were excluded from CAD by computed tomography angiography or magnetic resonance angiography or from individuals attending a routine health screening in the same hospital. The clinical and demographic characteristics of the population are shown in Table 2. CAD patients were much older, had a higher rate of smoking, higher BMI, TC, TG and LDL-C, and were more likely to suffer from diabetes, and to present with hypertension and dyslipidemia (Table 2).

\begin{tabular}{|c|c|c|c|}
\hline Characteristics & Case group $(N=236)$ & Control group $(N=278)$ & $\mathrm{P}$ \\
\hline Age (year) & $62.4 \pm 9.8$ & $60.3 \pm 10.2$ & 0.018 \\
\hline Gender (Male) [N (\%)] & $158(66.9)$ & $162(58.3)$ & $<0.001$ \\
\hline $\mathrm{BMI}\left(\mathrm{kg} / \mathrm{m}^{2}\right)$ & $24.6 \pm 2.78$ & $23.63 \pm 2.54$ & $<0.001$ \\
\hline Hypertension [N (\%)] & $141(59.7)$ & $116(41.7)$ & $<0.001$ \\
\hline Diabetes $[\mathrm{N}(\%)]$ & $84(35.6)$ & $62(22.3)$ & $<0.001$ \\
\hline Smoking [N (\%)] & $138(58.5)$ & $98(35.3)$ & $<0.001$ \\
\hline TG & $1.58 \pm 0.86$ & $1.26 \pm 0.76$ & $<0.001$ \\
\hline TC & $4.26 \pm 0.74$ & $3.94 \pm 0.82$ & $<0.001$ \\
\hline LDL-C & $2.49 \pm 0.62$ & $2.18 \pm 0.76$ & $<0.001$ \\
\hline HDL-C & $1.18 \pm 0.26$ & $1.41 \pm 0.31$ & $<0.001$ \\
\hline
\end{tabular}

$\mathrm{BMI}=$ body mass index.

\section{SNPs of the MEF2A gene and CAD risk}

Codominant, dominant, and recessive genetic models were applied to assess the association of rs325380, rs325381, rs325383, and rs897074 with CAD risk. Under the dominant model, GG+GT genotypes were associated with an increased CAD risk of rs325380. Under the codominant model, the "CC" genotype of rs 897074 was associated with an increased CAD risk. Similarly, under the dominant model, the CC+CT genotypes of rs897074 were associated with an increased CAD risk compared with the "T/T" genotype (Table 3). However, these values were not significant after Bonferroni's correction $\left(P_{\text {correction }}=0.05 / 7=0.007\right)$.

Table 3. Genotype distributions of SNPs and analysis of their association with CAD (adjusted by age and smoking rate).

\begin{tabular}{|c|c|c|c|c|c|c|}
\hline SNPs & Genotype & CAD & Control & Model & OR $(95 \% \mathrm{Cl})$ & $P$ value \\
\hline \multirow[t]{4}{*}{ rs325380 } & G/G & $102(43.4 \%)$ & $94(33.9 \%)$ & Codominant & $1.46(1.01-2.12)$ & 0.082 \\
\hline & $\mathrm{T} / \mathrm{G}$ & $110(46.8 \%)$ & $148(53.4 \%)$ & & $1.65(0.91-2.99)$ & \\
\hline & $T / T$ & $23(9.8 \%)$ & $35(12.6 \%)$ & Dominant & $1.49(1.04-2.14)$ & 0.028 \\
\hline & & & & Reccessive & $1.33(0.76-2.33)$ & 0.310 \\
\hline \multirow[t]{4}{*}{ rs325381 } & $T / T$ & $89(37.7 \%)$ & $84(30.2 \%)$ & Codominant & $1.34(0.91-1.97)$ & 0.150 \\
\hline & $\mathrm{A} / \mathrm{T}$ & $115(48.7 \%)$ & 145 (52.2\%) & & $1.62(0.94-2.77)$ & \\
\hline & $\mathrm{A} / \mathrm{A}$ & $32(13.6 \%)$ & 49 (17.6\%) & Dominant & $1.40(0.97-2.02)$ & 0.072 \\
\hline & & & & Reccessive & $1.36(0.84-2.22)$ & 0.210 \\
\hline \multirow[t]{4}{*}{ rs325383 } & $\mathrm{C} / \mathrm{C}$ & $93(39.6 \%)$ & $90(32.7 \%)$ & Codominant & $1.36(0.93-1.99)$ & 0.270 \\
\hline & $\mathrm{C} / \mathrm{T}$ & $111(47.2 \%)$ & 146 (53.1\%) & & $1.30(0.75-2.26)$ & \\
\hline & $T / T$ & $32(13.2 \%)$ & $49(14.2 \%)$ & Dominant & $1.35(0.94-1.94)$ & 0.110 \\
\hline & & & & Reccessive & $1.09(0.66-1.82)$ & 0.740 \\
\hline \multirow[t]{4}{*}{ rs897074 } & $\mathrm{C} / \mathrm{C}$ & $84(36.0 \%)$ & $73(26.4 \%)$ & Codominant & $1.47(0.93-1.99)$ & 0.038 \\
\hline & $\mathrm{C} / \mathrm{T}$ & $111(47.6 \%)$ & $141(51.1 \%)$ & & $1.87(1.12-3.12)$ & \\
\hline & $T / T$ & $38(16.3 \%)$ & $62(22.5 \%)$ & Dominant & $1.57(1.08-2.30)$ & 0.019 \\
\hline & & & & Reccessive & $1.48(0.94-2.32)$ & 0.084 \\
\hline
\end{tabular}

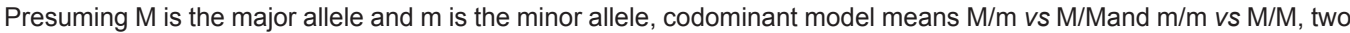
OR values were listed from top to bottom in the corresponding column; dominant model means $(\mathrm{m} / \mathrm{m}+\mathrm{M} / \mathrm{m})$ vs $M / M$; recessive model means $\mathrm{m} / \mathrm{m}$ vs $(\mathrm{M} / \mathrm{M}+\mathrm{M} / \mathrm{m})$; age, gender, and smoking status were adjusted for all models. 


\section{Haplotype of MEF2A and CAD risk}

LD block was formed by the Gabriel algorithm (Barrett et al., 2005) using SNP genotyping data in this study. The block consisted of two SNPs (rs325380 and rs325381). Three haplotypes (GT, TA, and GA) with a frequency over 0.01 were constructed. Haplotype TA carriers were determined to have a 1.34 -fold higher risk of CAD than GT carriers (Table 4).

\begin{tabular}{|c|c|c|c|c|c|}
\hline rs325380 & rs325381 & Frequencies in control group & Frequencies in CAD group & OR $(95 \% \mathrm{Cl})$ & $P$ value \\
\hline G & $\mathrm{T}$ & 0.6140 & 0.5553 & $1.00^{\mathrm{a}}$ & - \\
\hline $\mathrm{T}$ & $A$ & 0.3256 & 0.3845 & $1.34(1.02-1.76)$ & 0.039 \\
\hline G & $A$ & 0.0536 & 0.0526 & $1.08(0.61-1.92)$ & 0.800 \\
\hline
\end{tabular}

In this table, haplotypes with frequency over 0.01 were displayed. $\mathrm{CAD}=$ coronary artery disease; $\mathrm{OR}=$ odds ratio; $\mathrm{Cl}$

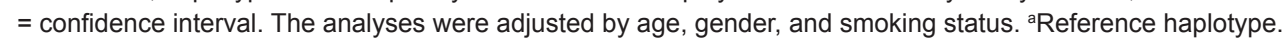

\section{DISCUSSION}

The relationship between MEF2A gene polymorphism and CAD has been controversial. To our knowledge, this is the first study to investigate the association between rs325380, rs325381, rs325383, and rs897074 in the 3'-UTR of the MEF2A gene and the risk of CAD in the Chinese Han population. After adjusting for confounding factors (age, gender, and smoking status), we found that rs325380 and rs897074 were associated with CAD risk. Bofferoni's correction, however, revealed that the association with CAD was not significant for any of the four target SNPs ( $P>$ 0.007). The genotype data from this study were further analyzed using the Haploview software; this assigned rs325380 and rs325381 to one LD block, and also revealed that carriers of the TA haplotype have a higher risk of CAD.

The polymorphisms located in the 3'-UTR might be involved in regulation of the MEF2A expression. Therefore, we used MirSNP (http://cmbi.bjmu.edu.cn/mirsnp) to identify microRNAbinding sites. The search shows that the G-to-T allele transversion in rs325380 may create a binding site in the MEF2A 3'-UTR for hsa-miR-412. Similarly, C-to-T allele transitions in rs897074 may create a binding for both hsa-miR-3065-3p and hsa-miR-634. The situation with the A-to-T allele transversion is more complex, as it may create some miR binding sites but destroy others. An in vitro study determined that miRNAs can inhibit skeletal muscle differentiation and cardiomyocyte growth by repressing MEF2A expression (Seok et al., 2011). Variants in the 3'-UTR, as well as a haplotype consisting of such variants identified in this study indicates that altered responses to miRNAs may contribute to pathologies associated with MEF2A. However, further studies of the effect that miRNA shave on MEF2A expression are required to elucidate the underlying mechanisms involved in CAD etiology. An earlier animal study found that MEF2A expression is post-transcriptionally repressed by its 3'-UTR (Black et al., 1997), and deregulation of this process might also contribute to CAD pathophysiology.

\section{Conflicts of interest}

The authors declare no conflict of interest. 


\section{REFERENCES}

Barrett JC, Fry B, Maller J and Daly MJ (2005). Haploview: analysis and visualization of LD and haplotype maps. Bioinformatics 21: 263-265.

Black BL, Lu J and Olson EN (1997). The MEF2A 3' untranslated region functions as a cis-acting translational repressor. Mol. Cell Biol. 17: 2756-2763.

Chen JM, Ferec C and Cooper DN (2006a). A systematic analysis of disease-associated variants in the 3 ' regulatory regions of human protein-coding genes I: general principles and overview. Hum. Genet. 120: 1-21.

Chen JM, Ferec C and Cooper DN (2006b). A systematic analysis of disease-associated variants in the $3^{\prime}$ regulatory regions of human protein-coding genes II: the importance of mRNA secondary structure in assessing the functionality of 3' UTR variants. Hum. Genet. 120: 301-333.

Dai DP, Zhou XY, Xiao Y, Xu F, et al. (2010). Structural changes in exon 11 of MEF2A are not related to sporadic coronary artery disease in Han Chinese population. Eur. J. Clin. Invest. 40: 669-677.

Dai Y, Zhang S and Wu W (2013). Analysis of MEF2A mutations in a Chinese population with premature coronary artery disease. Genet. Test Mol. Biomarkers 17: 352-355.

Elhawari S, Al-Boudari O, Muiya P, Khalak H, et al. (2010). A study of the role of the Myocyte-specific Enhancer Factor-2A gene in coronary artery disease. Atherosclerosis 209: 152-154.

Foroughmand AM, Shahbazi Z, Galehdari H, Purmahdi Borujeni M, et al. (2014). Association of MEF2A gene polymorphisms with coronary artery disease. Iran Red. Crescent Med. J. 16: e13533.

Guella I, Rimoldi V, Asselta R, Ardissino D, et al. (2009). Association and functional analyses of MEF2A as a susceptibility gene for premature myocardial infarction and coronary artery disease. Circ. Cardiovasc. Genet. 2: 165-172.

Juszczuk-Kubiak E, Starzynski RR, Wicinska K and Flisikowski K (2012). Promoter variant-dependent mRNA expression of the MEF2A in longissimus dorsi muscle in cattle. DNA Cell Biol. 31: 1131-1135.

Kangas-Kontio T, Huotari A, Ruotsalainen H, Herzig KH, et al. (2010). Genetic and environmental determinants of total and high-molecular weight adiponectin in families with low HDL-cholesterol and early onset coronary heart disease. Atherosclerosis 210: 479-485.

Kuersten S and Goodwin EB (2003).The power of the 3' UTR: translational control and development. Nat. Rev. Genet. 4: 626-637.

Liu Y, Niu W, Wu Z, Su X, et al. (2012). 2012 Variants in exon 11 of MEF2A gene and coronary artery disease: evidence from a case-control study, systematic review, and meta-analysis. PLoS One 7: e31406.

Seok HY, Tatsuguchi M, Callis TE, He A, et al. (2011). miR-155 inhibits expression of the MEF2A protein to repress skeletal muscle differentiation. J. Biol. Chem. 286: 35339-35346.

Shyu AB, Wilkinson MF and van Hoof A (2008). Messenger RNA regulation: to translate or to degrade. EMBO J. 27: 471-481.

Wang L, Fan C, Topol SE, Topol EJ, et al. (2003). Mutation of MEF2A in an inherited disorder with features of coronary artery disease. Science 302: 1578-1581. 